Gottfried Jetschke

Mathematik der Selbstorganisation 
Gottfried Jetschke

\section{Mathematik der Selbstorganisation}

Qualitative Theorie nichtlinearer dynamischer Systeme und gleichgewichtsferner Strukturen in Physik, Chemie und Biologie

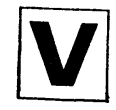

Friedr. Vieweg \& Sohn Braunschweig/Wiesbaden 


\section{CIP-Kurztitelaufnahme der Deutschen Bibliothek}

\section{Jetschke, Gottfried:}

Mathematik der Selbstorganisation : qualitative Theorie nichtlinearer dynamischer Systeme und gleichgewichtsferner Strukturen in Physik, Chemie und Biologie / Gottfried

Jetschke. - Braunschweig ; Wiesbaden : Vieweg, 1989

ISBN-13: 978-3-528-06346-7 e-ISBN-13: 978-3-322-85918-1

DOI: 10.1007/978-3-322-85918-1

1989

Alle Rechte vorbehalten

(C) VEB Deutscher Verlag der Wissenschaften, Berlin 1989

Softcover reprint of the hardcover 1st edition 1989

Lizenzausgabe für Friedr. Vieweg \& Sohn Verlagsgesellschaft mbH, Braunschweig, mit Genehmigung des VEB Deutscher Verlag der Wissenschaften Berlin/DDR 


\section{Vorwort}

Das Forschen nach den Ursachen der spontanen Strukturbildung in der unbelebten und der belebten Natur ist gegenwärtig ein faszinierendes und breit betriebenes Anliegen der modernen Physik, Chemie und Biologie. Die Entdeckung, daß in offenen gleichgewichtsfernen und deshalb nichtlinearen Systemen geordnete zeitliche, räumliche oder funktionale Strukturen als Folge kooperativer innerer Wechselwirkungen entstehen können, hat zu einer stürmischen Entwicklung auf diesem Gebiet geführt und auch die mathematische Forschung wesentlich befruchtet. Aber auch in vielen anderen Bereichen aus Naturwissenschaft und Technik wird das Wissen um Verhaltensweisen nichtlinearer dynamischer Systeme und deren Beherrschung heute unabdingbar.

Das vorliegende Lehrbuch ist aus Vorlesungen entstanden, die ich in den Jahren 1981-1986 an der Friedrich-Schiller-Universität Jena vor Physik- und Mathematikstudenten gehalten habe und will solche grundlegenden Kenntnisse einer modernen Theorie nichtlinearer Systeme vermitteln. Damit soll eine spürbare Lücke in der vorhandenen Lehrbuchliteratur geschlossen und ein Material bereitgestellt werden, das an anderen Einrichtungen Grundlage ähnlicher Kurse sein könnte.

Beim Aufbau des Buches habe ich die Darstellung der mathematischen Methoden zur Behandlung nichtlinearer autonomer dynamischer Systeme in den Vordergrund gerückt. Deshalb wird großer Wert auf eine möglichst präzise Formulierung von Begriffen und Sachverhalten gelegt, so daß die meisten Aussagen in Form mathematischer ,Sätze“ gegeben und weitgehend bewiesen werden. Die Beweise einiger tiefliegender Theoreme werden nur angedeutet, doch es dürfte insgesamt eine gut begründete und nachvollziehbare mathematische Theorie entstehen. Durchgängige physikalische Motivation bleibt das Problem der spontanen Strukturbildung, dem auch sämtliche Beispiele gewidmet sind. Zusammen mit der Einführung und dem ausführlichen thermodynamischen Anhang soll dies wesentlich dazu beitragen, daß zugleich die physikalische Theorie der Selbstorganisation in gleichgewichtsfernen Systemen erlernt wird.

Das Buch wendet sich vorrangig an theoretische Physiker sowie Chemiker, Biologen, Techniker und andere „Anwender“, aber auch an Mathematiker, denen die behandelten Methoden bzw. deren Anwendungen weniger geläufig sind. Den Physiker (und andere) möchte ich auffordern, sich nicht von der Art der Darstellung und der verwendeten modernen Sprechweise abschrecken zu lassen; viele Begriffe (z. B. Diffeo- 
morphismus, Phasenfluß) sind nur Abkürzungen für überschaubare Zusammenhänge. Vorausgesetzt werden neben dem Wissen um einfache Integrationsverfahren von Differentialgleichungen lediglich die Anfänge der Funktionalanalysis und ein Grundverständnis der Wahrscheinlichkeitstheorie. Dem ernsthaften Mathematiker bleibt es natürlich nicht erspart, sich die Details der Beweise in der angegebenen Literatur zu erarbeiten.

Den Kern des Buches bilden die Kapitel 1-5, in denen systematisch die qualitative Theorie nichtlinearer gewöhnlicher Differentialgleichungen in ein, zwei und mehr Dimensionen entwickelt wird. Zugleich werden zeitdiskrete Systeme diskutiert, die sonst verhältnismäßig selten behandelt werden, obwohl es in der Betrachtung viele Gemeinsamkeiten gibt. Einen breiten Raum habe ich dem Kapitel 5 über „Chaos“ gegeben, weil hier wichtige Ergebnïsse der gegenwärtigen Forschung vorgestellt werden, die von unmittelbarer praktischer Bedeutung sind. Das Kapitel 8 dehnt die Art der Untersuchung auf partielle. Differentialgleichungen aus, während die Kapitel 6 und 7 ergänzenden und systematisierenden Charakter tragen und für das erste Lesen nicht notwendig sind.

Einen zweiten Schwerpunkt bilden die Kapitel 9-11, die sich mit verschiedenen stochastischen Systemen, insbesondere stochastischen Differentialgleichungen beschäftigen. Dabei wird in Abschnitt 9.1. die in der Mathematik übliche maßtheoretische Formulierung eingeführt, um anschließend diskrete und stetige Verteilungen bzw. Zufallsgrößen einheitlich behandeln zu können. Etwas anspruchsvoller ist das Kapitel 13 über stochastische partielle Differentialgleichungen (das die Kenntnis von Kapitel 8 voraussetzt), es beruht im wesentlichen auf Forschungsergebnissen des Autors.

Zwei wichtige Fragestellungen werden nicht (oder fast nicht) behandelt, nämlich analytische Näherungsverfahren sowie Methoden zur numerischen Lösung von Differentialgleichungen bzw. deren Simulation. Hier muß auf die entsprechende Literatur verwiesen werden.

Dem Leser, der das Buch nicht von Anfang bis Ende durcharbeiten möchte und sich nur für die wichtigsten mathematischen Aussagen interessiert, möchte ich unbedingt die Kapitel 1, 3, 5, 8 sowie 10 empfehlen. Wer dagegen vorrangig die physikalischen Anwendungen studieren will, der sollte in folgender Weise vorgehen: Einführung (E.), thermodynamische Grundlagen (A.3.), mathematische Methoden (1., 3.1. -3.3., 4.1., 5., 8.2., 9.), physikalische Systeme (2., 3.1.), chemische Systeme (2.2., 3.6., 6.2., 8.3., 8.4.), biologische Systeme (2.2., 3.6., A.2.4., 4.3.), stochastische Modellierung (10.1.3., 10.2.3., 10.3., 11., 13.3.).

Kommentierende Textstellen, Nebengedanken oder für das erste Lesen weniger wichtige Passagen sind in Kleindruck gesetzt, ebenso die meisten Beispiele, sofern sie nicht grundsätzlicher Natur sind. Der Leser sollte sich aber auf jeden Fall um die selbständige Lösung der zahlreichen Aufgaben bemühen, um seine Fähigkeiten zur Anwendung der erworbenen Kenntnisse zu überprüfen. Die Hinweise im Anhang sind dabei als Hilfestellung und zum nachträglichen Vergleich gedacht.

Noch einige Bemerkungen zur Notation, die an manchen Stellen von der in der Physik üblichen abweicht: Vektoren werden generell nicht durch Pfeile oder Fettdruck 
hervorgehoben, weil ihr Charakter in der Regel aus dem Zusammenhang eindeutig hervorgeht. Elemente des $n$-dimensionalen Raumes $\mathbb{R}^{n}$ werden mit $x=\left(x_{1}, \ldots, x_{n}\right)$ bezeichnet, analog Vektorfelder mit $f=\left(f_{1}, \ldots, f_{n}\right)$. Im Falle $n=2$ bzw. $n=3$ wird häufig die indexsparende Schreibweise $(x, y)$ bzw. $(x, y, z)$ verwendet. Die JacobiMatrix einer Abbildung $f: \mathbb{R}^{n} \rightarrow \mathbb{R}^{m}$ wird kurz mit $f^{\prime}(x)$ bezeichnet. Wo es nicht nötig ist, werden Spalten- und Zeilenvektoren nicht unterschieden. Das Skalarprodukt zweier Vektoren $a$ und $b$ wird mit $(a, b)$ abgekürzt. Ein Punkt über einer Variablen bedeutet stets die (totale) Ableitung nach der Zeit (also $\dot{x} \equiv d x(t) / d t)$.

Definitionen, Sätze, Beispiele und Aufgaben sind innerhalb jedes zweiziffrigen Abschnittes - dreiziffrige Unterabschnitte, wie 1.4.1., dienen nur der übersichtlicheren Gliederung - fortlaufend numeriert und benannt, sie werden außerhalb des jeweiligen Abschnittes in der Form Definition (Satz, ...) 1.4./2 zitiert. Formeln sind jeweils im gesamten Kapitel durchgängig numeriert (z. B. Gleichung (1.13)).

Danken möchte ich zuerst meinen akademischen Lehrern, Herrn Dr. G. NeUgebauer und Herrn Prof. H. Trieber, die mich direkt oder indirekt mit den dargestellten Ideen und Methoden vertraut gemacht haben. Ebenfalls danken möchte ich all meinen Hörern und Kollegen, namentlich Herrn Prof. K.-H. FichtneR, für ihre kritischen Hinweise zur Vorlesung und zu Teilen des Manuskripts. Ganz besonderer Dank gebührt meiner Studentin Frl. CH. Strewe für die mehrmalige aufmerksame Durchsicht des Manuskripts während seiner Entstehung sowie Frau G. GIRLICH für die sorgfältige Reinschrift. Für die Anfertigung der Computerrechnungen zu den Abbildungen 63, 69, 73 und 144 bin ich Herrn Dr. T. Wolf zu Dank verpflichtet. Dem VEB Deutscher Verlag der Wissenschaften und der verantwortlichen Lektorin, Frau K. BRATz, danke ich für die aufgeschlossene Zusammenarbeit. 


\section{Inhaltsverzeichnis}

E. Einführung .......................... . 13

E.1. Ordnung und Selbstorganisation . . . . . . . . . . . . . . . . . . . . 13

E.2. Selbsterregte Schwingungen einer gestrichenen Saite . . . . . . . . . . . . . . . . 15

E.3. Dissipative Strukturen . . . . . . . . . . . . . . . . . . . . . . . . . 19

1. Deterministische dynamische Systeme . . . . . . . . . . . . . . . . . . 24

1.1. Phasenfluß . . . . . . . . . . . . . . . . . . . . . 24

1.2. Gewöhnliche Differentialgleichungen . . . . . . . . . . . . . . . . . . . 25

1.2.1. Lösung einer Differentialgleichung . . . . . . . . . . . . . . . . . . . 25

1.2.2. Existenz und Eindeutigkeit . . . . . . . . . . . . . . . . . . . . . . . . 28

1.2.3. Fortsetzbarkeit . . . . . . . . . . . . . . . . . . . . . . . . . . 30

1.3. Lineare Differentialgleichungen mit konstanten Koeffizienten . . . . . . . . . 31

1.3.1. Allgemeine Lösung von $\dot{x}=A x$. . . . . . . . . . . . . . . . . . . . . . . . . 31

1.3.2. Berechnung von $\mathrm{e}^{t A}$. . . . . . . . . . . . . . . . . . . . . . . . . . . . . 33

1.3.3. Topologische Typen linearer Flüsse . . . . . . . . . . . . . . . . . . . . 38

1.4. Stabilität von Fixpunkten . . . . . . . . . . . . . . . . . . . . . . . $4 \mathbf{4 1}$

1.4.1. Methode der Linearisierung. . . . . . . . . . . . . . . . . . . . . . . . . . . . . 43

1.4.2. Methode der Ljapunow-Funktion . . . . . . . . . . . . . . . . . . . . . . . . 48

1.5. Grenzmengen und Attraktoren . . . . . . . . . . . . . . . . . . . . . . 52

1.6. Zeitdiskrete Systeme (iterierte Abbildungen) . . . . . . . . . . . . . . . . . . . . . . . . 58

1.7. Strukturelle Stabilität . . . . . . . . . . . . . . . . . . . . . 65

2. Systeme mit einem Freiheitsgrad . . . . . . . . . . . . . . . . . . . . 68

2.1. Allgemeine Eigenschaften . . . . . . . . . . . . . . . . . . . . . . . 68

2.2. Weitere Beispiele . . . . . . . . . . . . . . . . . . . . . 70

3. Systeme mit zwei Freiheitsgraden . . . . . . . . . . . . . . . . . . . . 75

3.1. Multistabilität . . . . . . . . . . . . . . . . . . . . 75

3.2. Grenzzyklen. Satz von Poincaré . . . . . . . . . . . . . . . . . . . . 81

3.3. Wiederkehrabbildung . . . . . . . . . . . . . . . . . . . . . . . . . 84

3.4. Van der Polsche Differentialgleichung . . . . . . . . . . . . . . . . . . . . 87

3.5. Mittelungsverfahren . . . . . . . . . . . . . . . . . . . . . . . . . . 92

3.6. Weitere Beispiele . . . . . . . . . . . . . . . . . . . . . . . . . . . 95

3.7. Poincaré-Index . . . . . . . . . . . . . . . . . . . . . . . . . . 100

4. Systeme mit mehr als zwei Freiheitsgraden . . . . . . . . . . . . . . . 103

4.1. Invariante Tori . . . . . . . . . . . . . . . . . . . . . . . . . . . . 103

4.2. Elimination schneller Variabler . . . . . . . . . . . . . . . . . 105

4.3. Selektion und Evolution . . . . . . . . . . . . . . . . . . . . . . . . . . . . . . . . . . 108 
5. Chaotische Attraktoren

5.1. Chaos in zeitdiskreten Systemen . . . . . . . . . . . . . . . . . . . . 112

5.1.1. Stückweise lineare Abbildungen . . . . . . . . . . . . . . . . . . . . . 113

5.1.2. Parabel-Abbildung . . . . . . . . . . . . . . . . . . . . . . . . . . 117

5.1.3. Hénon-Abbildung . . . . . . . . . . . . . . . . . . . . . . . . . . . 128

5.2. Chaos bei Differentialgleichungen . . . . . . . . . . . . . . . . . . . . . 129

5.2.1. Lorenz-Attraktor . . . . . . . . . . . . . . . . . . . . . . . . . . . 130

5.2.2. Ergänzungen . . . . . . . . . . . . . . . . . . . . . . . . . . 136

5.3. $\omega$-Grenzmengen und invariante Verteilungen . . . . . . . . . . . . . . . 138

5.4. Eigenschaften chaotischer Attraktoren . . . . . . . . . . . . . . . . . . 143

5.4.1. Ljapunow-Exponenten . . . . . . . . . . . . . . . . . . . . . . . . . 143

5.4.2. Weitere Eigenschaften . . . . . . . . . . . . . . . . . . . . 150

6. Bifurkationstheorie . . . . . . . . . . . . . . . . . . . . 155

6.1. Zentrale Mannigfaltigkeit . . . . . . . . . . . . . . . . . . . 155

6.2. Bifurkationen von Fixpunkten einparametriger Differentialgleichungen . . . 158

6.3. Bifurkationen von Fixpunkten einparametriger Abbildungen . . . . . . . . 168

7. Katastrophentheorie . . . . . . . . . . . . . . . . . . . . . . . . . 171

7.1. Einführung. . . . . . . . . . . . . . . . . . . . . . 171

7.2. Falten und Spitzen . . . . . . . . . . . . . . . . . . . . . . 176

7.3. Elementare Katastrophen . . . . . . . . . . . . . . . . . . . . . . . 178

8. Reaktions-Diffusions-Systeme . . . . . . . . . . . . . . . . . . . 183

8.1. Grundgleichung . . . . . . . . . . . . . . . . . . . . . . . . . . . . 183

8.2. Fixpunkte und deren Stabilität . . . . . . . . . . . . . . . . . . . . . 184

8.3. Kubische Nichtlinearität und Diffusion . . . . . . . . . . . . . . . . . 187

8.4. Brüsselator mit Diffusion . . . . . . . . . . . . . . . . . . . . . . . 193

9. Stochastische dynamische Systeme . . . . . . . . . . . . . . . . . . 199

9.1. Wahrscheinlichkeitstheoretische Grundbegriffe . . . . . . . . . . . . 199

9.2. Stochastische Prozesse . . . . . . . . . . . . . . . . . . . . . . . . 205

9.3. Markow-Prozesse . . . . . . . . . . . . . . . . . . . . . . . . 207

10. Stochastische Differentialgleichungen . . . . . . . . . . . . . 216

10.1. Additives weißes Rauschen . . . . . . . . . . . . . . . . . . . . . . . 216

10.1.1. Modell und Lösungsbegriff . . . . . . . . . . . . . . . . . . . . . . . 216

10.1.2. Markow-Eigenschaft. Invariante Verteilung . . . . . . . . . . . . . . . 218

10.1.3. Beispiele . . . . . . . . . . . . . . . . . . . . . . . . . . . . . . 223

10.2. Multiplikatives weißes Rauschen . . . . . . . . . . . . . . . . . . . . 228

10.2.1. Lösung einer stochastischen Differentialgleichung . . . . . . . . . . . . . . . 228

10.2.2. Markow-Eigenschaft. Randverhalten. Invariante Verteilung . . . . . . . . 231

10.2.3. Rauschinduzierte Übergänge . . . . . . . . . . . . . . . . . . . . . . 235

10.3. Farbiges Rauschen . . . . . . . . . . . . . . . . . . . . . . . . . . 238

11. Geburts- und Todesprozesse . . . . . . . . . . . . . . . . . . . . . . . 242

11.1. Modell und Grundgleichungen . . . . . . . . . . . . . . . . . . . . . 242

11.2. Invariante Verteilung . . . . . . . . . . . . . . . . . . . . . . . . . 246 
12. Zeitdiskrete Systeme mit Rauschen . . . . . . . . . . . . . . . . . 252

13. Stochastische partielle Differentialgleichungen . . . . . . . . . . . . . . 255

13.1. Modell und Lösungsbegriff . . . . . . . . . . . . . . . . . . . . . . 255

13.2. Markow-Charakter und invariante Verteilung . . . . . . . . . . . . . . . 258

13.3. Wahrscheinlichste Zustände und Tunnelverhalten . . . . . . . . . . . . . 262

A. Anhang. . . . . . . . . . . . . . . . . . . . 266

A.1. Mathematische Modellbildung . . . . . . . . . . . . . . . . . . 266

A.2. Einzelwissenschaftliche Ergänzungen . . . . . . . . . . . . . . . . . . 268

A.2.1. Mechanische Systeme . . . . . . . . . . . . . . . . . . . . . . . . 268

A.2.2. Elektrische Systeme . . . . . . . . . . . . . . . . . . . . . . . . . . 269

A.2.3. Chemische Systeme . . . . . . . . . . . . . . . . . . . . . . . . . . 272

A.2.4. Biologische Systeme . . . . . . . . . . . . . . . . . . . . . . . . . . 276

A.3. Thermodynamische Grundlagen . . . . . . . . . . . . . . . . . . . . . 280

A.3.1. Systeme im thermodynamischen Gleichgewicht . . . . . . . . . . . . . . 280

A.3.2. Nichtgleichgewichtssysteme. . . . . . . . . . . . . . . . . . . . . . . 284

A.3.3. Thermodynamische Stabilitätstheorie . . . . . . . . . . . . . . . . . . 290

A.4. Synergetik . . . . . . . . . . . . . . . . . . . . . . . 296

Lösungen der Aufgaben . . . . . . . . . . . . . . . . . . . . . . . . 301

Weiterführende Literatur . . . . . . . . . . . . . . . . . . . . 325

Abbildungsnachweis . . . . . . . . . . . . . . . . . . . 329

Sachverzeichnis . . . . . . . . . . . . . . . . . . . . 330 